\title{
O Impacto da COVID-19 na População Idosa em Portugal: Resultados do Survey of Health, Ageing and Retirement (SHARE)
}

\section{The Impact of COVID-19 in Older People in Portugal: Results from the Survey of Health, Ageing and Retirement (SHARE)}

\author{
Filipa NOVAIS $\square^{1,2}$, Catarina CORDEIRO ${ }^{1,2}$, Pedro CÂMARA PESTANA ${ }^{1,2}$, Beatriz CÔRTE-REAL ${ }^{1,2}$, \\ Teresa REYNOLDS SOUSA ${ }^{1,2}$, Alice DELERUE MATOS ${ }^{3}$, Diogo TELLES-CORREIA ${ }^{1,2}$ \\ Acta Med Port 2021 Nov;34(11):761-766 - https://doi.org/10.20344/amp.16209
}

\section{RESUMO}

Introdução: Em março de 2020, a COVID-19 foi declarada pandemia pela Organização Mundial da Saúde e Portugal registou os seus primeiros casos. Este estudo visou determinar o impacto da COVID-19 na população portuguesa maior de 60 anos.

Material e Métodos: Trata-se de um estudo observacional transversal que analisa várias variáveis de saúde, comportamentais, sociais e económicas. Utilizaram-se dados do projecto Survey of Health, Ageing and Retirement (SHARE 8: COVID-19 Survey), seleccionando uma amostra de 1080 portugueses não institucionalizados e maiores de 60 anos.

Resultados: A amostra englobou 605 mulheres (56\%) e 475 homens (44\%), com idade média de $70 \pm 9,1$ anos. No total, $80 \%$ dos participantes notou aumento da ansiedade, $73 \%$ sentiu-se mais deprimido e $30 \%$ considerou que o seu sono piorou em relação ao período anterior à pandemia. Não houve diferenças estatisticamente significativas entre os sexos ou as duas faixas etárias analisadas ( 60 - 74 e $\geq 75$ anos) quanto à incidência destas alterações. No respeitante a saídas de casa, apenas $23 \%$ dos entrevistados manteve os hábitos de passeio e $8 \%$ manteve as visitas a familiares no seu padrão habitual. Relativamente ao acesso a cuidados médicos, a $8 \%$ foi negado algum tratamento e $56 \%$ queixou-se do adiamento dos cuidados. Apenas $13 \%$ referiu um agravamento do seu estado de saúde durante a pandemia.

Discussão: Este trabalho mostra que esta pandemia impactou a população portuguesa com mais de 60 anos, o que está em linha com o reportado noutros estudos internacionais. Os hábitos alteraram-se, e a ansiedade e depressão aumentaram. Apesar da deterioração dos cuidados de saúde, a maioria dos participantes não notou agravamento do seu estado de saúde.

Conclusão: Em conclusão, a pandemia por COVID-19 teve um impacto significativo na população idosa particularmente no que concerne à sua saúde mental.

Palavras-chave: Avaliação Geriátrica; COVID-19; Idoso; Portugal; SARS-CoV-2; Saúde Mental

\section{ABSTRACT}

Introduction: In March 2020, the World Health Organization declared COVID-19 as a pandemic, and Portugal reported its initial cases. In this study, we aimed to determine the impact of COVID-19 on Portuguese individuals aged over 60 years old.

Material and Methods: We performed a cross-sectional study using data from the Survey of Health, Ageing and Retirement in Europe (SHARE 8: COVID-19 Survey). We selected a sample of 1080 noninstitutional Portuguese individuals aged $\geq 60$ years.

Results: The study sample consisted of $605(56 \%)$ women and $475(44 \%)$ men, with a mean age of $70 \pm 9.1$ years. In total, $80 \%$ of the participants experienced higher levels of anxiety, $73 \%$ felt more depressed and $30 \%$ experienced additional sleep problems compared to the period before the pandemic. Interestingly, there were no statistically significant differences between the sexes or the two selected age groups (60 - 74 and over 75 years old) regarding the incidence of these changes. Only $23 \%$, of those that were interviewed maintained their walking routines. In addition, only $8 \%$ of the participants continued visiting family members as frequently as before. While $8 \%$ of the participants were refused some form of medical treatment, $56 \%$ claimed that they experienced healthcare delays. However only $15 \%$ of the participants reported that their health status worsened during the pandemic.

Discussion: The pandemic has had a significant impact on Portuguese individuals aged $\geq 60$ years; which is in agreement with the findings of previous international studies. It changed the participants' routines and increased their anxiety and depression levels. Despite the deterioration of healthcare services, most participants did not experience worsening of their health status.

Conclusion: In conclusion, a COVID-19 pandemic had a significant impact on the elderly population, particularly regarding their menta health.

Keywords: Aged; COVID-19; Geriatric Assessment; Mental Health; Portugal SARS-CoV-2

\section{INTRODUÇÃO}

Em março de 2020, a Organização Mundial de Saúde (OMS) declarou a COVID-19 uma pandemia, ${ }^{1}$ e foi reportado o primeiro caso em Portugal. $^{2}$ Desde então, foram apli-

cadas restrições para conter a propagação do novo coronavírus. Durante dois meses (até meados de maio de 2020), vigorou o confinamento geral, com dever de recolhimento

1. Departamento de Neurociências e Saúde Mental. Departamento de Psiquiatria. Hospital de Santa Maria. Centro Hospitalar e Universitário de Lisboa Norte. Lisboa. Portugal.

2. Departamento de Psiquiatria. Faculdade de Medicina. Universidade de Lisboa. Lisboa. Portugal.

3. Departamento de Sociologia. Instituto de Ciências Sociais da Universidade do Minho. Braga. Portugal.

$\triangle$ Autor correspondente: Filipa Novais. fnovais@campus.ul.pt

Recebido: 16 de março de 2021 - Aceite: 22 de junho de 2021 - Online issue published: 02 de novembro de 2021

Copyright $\odot$ Ordem dos Médicos 2021 
em casa, encerramento de escolas, obrigação de teletrabaIho quando aplicável, encerramento dos estabelecimentos de comércio e restauração, entre outros. Em maio e junho do mesmo ano, deu-se um progressivo desconfinamento e entre julho e agosto não se verificaram restrições significativas. Entre junho e agosto de 2020, período abarcado neste trabalho, os números diários de novos casos de infeção rondaram os $200-350 .^{3}$ Com tudo isto, verificou-se uma crise nos sectores da saúde, social e económico, com profundo impacto sobre múltiplos níveis da sociedade e dimensões ainda por elucidar. ${ }^{4}$

Os efeitos psicológicos decorrentes da pandemia, agravados pelos confinamentos, são múltiplos, tais como depressão, ansiedade, irritabilidade, sintomas de pós-stress traumático. ${ }^{5} \mathrm{~A}$ maior parte dos estudos documenta taxas de ansiedade e depressão associados ao período COVID, mas não aborda as suas variações em relação ao período pré-pandémico. Uma meta-análise mostrou taxas de prevalência de ansiedade, depressão e alterações do sono de $33 \%, 28 \%$ e $32 \%$, respetivamente, o que significa um aumento das suas prevalências em relação ao previamente reportado. ${ }^{6}$ Um estudo alemão mostrou uma elevação das taxas de prevalência de ansiedade, depressão e distress psicológico durante o período pandémico. ${ }^{7}$ Outro, que teve por base a população de Hong Kong, reporta que $25 \%$ dos inquiridos afirmaram ter notado um agravamento da sua saúde mental. ${ }^{8}$ Curiosamente, um estudo norte-americano mostrou uma associação entre a instauração de medidas restritivas e uma diminuição da pesquisa de termos ligados à saúde mental nos motores de busca. ${ }^{9}$ Adicionalmente, a separação de familiares por períodos desconhecidos, a incerteza proveniente de mensagens contraditórias das autoridades de saúde e a sensação de perda de controlo, levam ao sentimento de aprisionamento que acarreta efeitos dramáticos para o bem-estar dos cidadãos. ${ }^{10}$

Todas as faixas etárias foram atingidas negativamente por este fenómeno, ${ }^{11,12}$ mas verificou-se um impacto maior nalguns grupos da população portuguesa, ${ }^{13}$ em particular as pessoas mais velhas, muitas vezes com dificuldades na adaptação ao mundo digital. Nestes casos, a exclusão social torna-se mais relevante e pode dar origem a um sentimento de dupla exclusão. ${ }^{14}$ Além disso, dada a maior mortalidade nas faixas etárias mais adiantadas, ${ }^{15}$ importa perceber o impacto da pandemia sobre estas. Da informação disponível, sabemos que o impacto é reconhecidamente superior em pessoas com patologia prévia. ${ }^{16}$

Assim, e sabendo a importância que as quarentenas têm na gestão desta pandemia, é importante avaliar de que modo os diferentes parâmetros da vida quotidiana, são impactados por esta situação, em particular nesta faixa etária, por forma a construir alternativas e recomendações dirigidas à população portuguesa, e em especial à população mais afetada em termos de mortalidade.

Neste contexto, o objetivo deste trabalho é caraterizar o impacto da pandemia por COVID-19 em pessoas com idade superior a 60 anos em Portugal.

\section{MATERIAL E MÉTODOS}

Neste estudo transversal utilizaram-se dados de uma amostra representativa da população portuguesa não institucionalizada e com mais de 60 anos, provenientes da Survey of Health, Ageing and Health (SHARE). Em relação à amostra colhida em Portugal, esta teve como base de amostragem o registo de utentes do Sistema Nacional de Saúde (SNS), tendo sido selecionados aleatoriamente residentes que falam português, nascidos até 1960 (para as vagas 4 , e 6 a 8), assim como, os seus cônjuges/companheiros, independentemente da idade destes últimos indivíduos.

Em relação à dimensão alvo da amostra, esta foi de 2000 indivíduos, tendo-se considerado uma taxa de resposta esperada de $60 \%$, uma proporção esperada de unidades não amostráveis de $10 \%$ e a realização de duas entrevistas em cerca de $50 \%$ dos agregados, pelo que a dimensão da amostra bruta é de 2507 indivíduos.

Quanto ao desenho da amostra, este compreendeu cinco etapas após a determinação de 22 sub-regiões do continente e ilhas, com a seleção de quatro dígitos do código postal na primeira etapa, das freguesias na segunda etapa, dos sete dígitos do código postal na terceira etapa, dos endereços na quarta etapa, e verificação da elegibilidade pela idade na quinta etapa.

Os dados foram recolhidos por meio de uma entrevista telefónica assistida por computador, dos quais foram selecionados apenas os participantes capazes de falar em língua portuguesa, incluídos na última vaga de colheita de dados. A informação relativa à oitava vaga a nível europeu (quarta em Portugal), foi colhida entre 11 de junho e 10 de agosto de 2020, inclui dados acerca da infeção por SARS-CoV-2 do próprio e de familiares, saúde global e psicológica, cuidados hospitalares, dados financeiros, laborais e sociais.

Nestas entrevistas aplicou-se o questionário disponível em http://www.share-project.pt/fileadmin/user_upload/sharew8_corona_questionnaire_pt_PT_20200601.pdf.

Em Portugal, a Universidade do Minho e a Universidade Nova de Lisboa, em cooperação com o SHARE- ERIC, foram os responsáveis pela implementação deste projeto.

Foi obtido consentimento oral por parte de todos os participantes.

A aprovação da vaga 8 foi pedida e obtida do Conselho de Ética da Sociedade Max Planck. Em Portugal, a colheita de dados foi aprovada pela Comissão de Ética para a Investigação em Ciências Sociais e Humanas (CEICSH) da Universidade do Minho

Os investigadores obtiveram acesso aos dados do SHARE mediante registo e declaração de compromisso ético.

Outros detalhes acerca desta base de dados podem ser acedidos online (www.share-project.pt). ${ }^{17,18}$

\section{Análise estatística}

A análise estatística foi realizada no software Stata (versão 14.2; StataCorp, Texas, EUA). As estatísticas descritivas foram apresentadas como proporções (\%) para 
variáveis dicotómicas, média e desvio padrão para as variáveis contínuas. Foi feita a calibração da amostra de acordo com a ponderação atribuída a cada indivíduo. Foram selecionados para este trabalho indivíduos com 60 ou mais anos, uma vez que a determinação da amostra representativa inicial de 50 e mais anos foi feita há 10 anos (2010/2011) e o painel não foi renovado desde então, pelo que representa apenas essa população.

Neste trabalho, foram analisadas as seguintes variáveis provenientes do questionário: perceção acerca do estado de saúde desde o início da pandemia (melhor/pior/ igual), saídas de casa desde o início da pandemia ( $\mathrm{sim} /$ não), agravamento da ansiedade ou sentimentos de nervosismo (mais/menos/igual), tristeza ou depressão (mais/ menos/igual), sentimentos de solidão (mais/menos/igual) ou dificuldades do sono desde o início da pandemia (mais/ menos/igual), teste positivos para SARS-CoV-2 do próprio (sim/não), internamento do próprio por SARS-CoV-2 (sim/ não), sintomas compatíveis com infeção por Coronavírus tais como tosse, febre ou dificuldade respiratória ( $\operatorname{sim} /$ não), mortes de 'alguém próximo' por SARS-CoV-2 (sim/não), adiamento, recusa na obtenção ou abandono pelo próprio de tratamentos médicos desde o início da pandemia por medo de ficar infetado (sim/não), desemprego que o próprio atribui à crise pandémica ( $\operatorname{sim} /$ não) e dificuldades económicas desde o início da pandemia, avaliadas através da questão "Pensando no rendimento mensal total do senhor(a) e das pessoas que vivem consigo, desde o início da epidemia do Coronavírus, diria que o dinheiro chega até ao fim do mês com grande dificuldade, com alguma dificuldade, com alguma facilidade ou facilmente?" (com grande dificuldade/com alguma dificuldade/com alguma facilidade/ facilmente).

Após esta análise descritiva inicial, dividiu-se a amostra por sexos e faixas etárias (60 - 74 anos e mais de 75 anos, de acordo com definição previamente proposta) ${ }^{19}$ e a comparação das variáveis anteriores de acordo com estes grupos, utilizando testes de qui-quadrado ou de Fisher para dados não-paramétricos.

O valor de $p \leq 0,05$ foi considerado estatisticamente significativo.

\section{RESULTADOS}

A partir de uma amostra inicial de 1652 pessoas foram excluídas 445 pessoas por terem idade inferior a 60 anos e 127 por falta de dados. Assim, 1080 participantes foram incluídos neste estudo, dos quais 605 eram mulheres (56\%) e 475 homens (44\%). Foram incluídos participantes entre os 60 e os 97 anos com uma média de idades de $70 \pm 9.1$ anos. Foram incluídas 594 pessoas (55\%) entre os 60 e os 69 anos, $288(27 \%)$ entre os 70 e os 79 anos, $163(15 \%)$ entre os 80 e os 89 anos e 35 (3\%) com mais de 90 anos.

Destes, apenas quatro das pessoas que indicaram ter tido ou conhecer alguém que tenha tido sintomas compatíveis com infeção por coronavírus (135 pessoas), indicaram ter tido um teste positivo para SARS-CoV-2, e duas pessoas tiveram necessidade de hospitalização. Contudo, 10 participantes terão tido sintomas compatíveis com infeção por coronavírus (tosse, febre ou dificuldades respiratórias) e 25 referiram que 'alguém próximo' terá falecido devida a infeção por coronavírus. Desde o início da pandemia, a maioria da população inquirida (83\%), considerou que o seu estado de saúde não se alterou e 15\% considerou que o seu estado de saúde piorou.

Dezasseis por cento dos inquiridos refere não ter saído de casa desde o início da pandemia e só $23 \%$ mantiveram os mesmos hábitos de sair de casa para fazer uma caminhada, tal como antes da pandemia. Vinte e nove por cento não voltaram a sair para fazer uma caminhada e 35\% dos inquiridos reduziu estes hábitos. Apenas $8 \%$ dos inquiridos mantiveram as visitas a familiares com a mesma regularidade e mais de $80 \%$ deixou de visitar ou visitou familiares com menos regularidade.

Oitenta por cento referiram um aumento da ansiedade ou nervosismo desde o início da pandemia e só $19 \%$ considerou não existirem alterações. Setenta e três por cento reconheceram sentir-se mais tristes ou deprimidos desde o início da pandemia e $27 \%$ declararam não terem existido alterações quanto ao seu humor. Quanto a dificuldades do sono, $30 \%$ consideraram que o seu sono piorou desde o início da pandemia e $70 \%$ acreditam que o seu sono não sofreu alterações. Quanto a sentimentos de solidão, 31\% dos participantes refere sentir-se mais só desde o início da pandemia.

Quanto à perceção acerca dos cuidados médicos durante este período, $12 \%$ desistiram de algum cuidado médico por medo de serem infetados com o novo coronavírus. Mais de metade desta amostra (56\%) referiu que houve um atraso nos seus tratamentos devido à pandemia. Em $8 \%$ dos casos esses tratamentos terão sido mesmo negados.

Trinta por cento dos participantes que estavam a trabaIhar perderam o emprego devido à pandemia e 59\% desta população referiu ter tido dificuldade em que o dinheiro chegasse até ao final do mês.

\section{Comparação entre sexos (Tabela 1)}

Comparando homens e mulheres, verifica-se que estas referiram com maior frequência um agravamento do seu estado de saúde desde o início da pandemia $(66 \% ; p<0,05)$; comparativamente aos homens também referiram mais frequentemente não terem saído de casa desde o início da pandemia $(67 \% ; p<0,05)$; abandonaram também mais frequentemente as consultas ou tratamentos em curso $(71 \%$; $p<0,05)$ e tiveram mais dificuldades em conseguir que os seus rendimentos fossem suficientes para cobrir as suas despesas até ao final do mês $(66 \% ; p<0,05)$.

\section{Comparação de acordo com o grupo etário (Tabela 2)}

Como único resultado estatisticamente significativo observou-se, entre os que anteriormente trabalhavam, que no grupo etário dos 60 aos 74 anos uma percentagem maior de participantes $(60 \%)$ refere ter perdido o emprego ou entrado em lay-off devido à pandemia $(p<0,05)$ em comparação com o grupo etário dos maiores de 75 anos. 
Tabela 1 - Análise de dados (por sexo) relativos à vivência das pessoas com idade superior a 60 anos durante a pandemia

\begin{tabular}{|c|c|c|c|}
\hline & Homens & Mulheres & $p$ \\
\hline Agravamento do estado de saúde desde o início da pandemia, n (\%) & $45(34)$ & $89(66)$ & $0,008^{*}$ \\
\hline Teste positivo para COVID-19 & $1(25)$ & $3(75)$ & 0,390 \\
\hline Hospitalização por COVID-19 & $0(0)$ & $2(100)$ & 0,640 \\
\hline Morte de alguém próximo por COVID-19 & $13(52)$ & $12(48)$ & 0,425 \\
\hline Sintomas compatíveis com infeção & $5(50)$ & $5(50)$ & 0,385 \\
\hline Nunca ter saído de casa desde o início da pandemia & $67(33)$ & $134(67)$ & $0,001^{*}$ \\
\hline Agravamento da ansiedade desde o início da pandemia & $125(35)$ & $236(65)$ & 0,097 \\
\hline Agravamento da tristeza desde o início da pandemia & $94(30)$ & $217(70)$ & 0,455 \\
\hline Agravamento de problemas do sono desde o início da pandemia & $32(28)$ & $83(72)$ & 0,191 \\
\hline Adiamento de consultas devido à pandemia & $220(43)$ & $294(57)$ & 0,385 \\
\hline Recusa de consulta médicos devido à pandemia pelos serviços & $32(42)$ & $44(58)$ & 0,721 \\
\hline Abandono de cuidados de saúde pelo próprio & $32(29)$ & $78(71)$ & $0,001^{*}$ \\
\hline Desemprego ou lay-off & $14(39)$ & $22(61)$ & 0,344 \\
\hline Dificuldades em realizar pagamentos até ao fim do mês & $123(34)$ & $244(66)$ & $0,011^{*}$ \\
\hline
\end{tabular}

${ }^{*}$ Resultados estatisticamente significativos $(p<0,05)$

Tabela 2 - Análise de dados (por faixa etária) relativos à vivência das pessoas com idade superior a 60 anos durante a pandemia

\begin{tabular}{|c|c|c|c|}
\hline & $\begin{array}{c}60-74 \text { anos } \\
(\mathrm{n}=756)\end{array}$ & $\begin{array}{c}75 \text { anos ou mais } \\
(n=324)\end{array}$ & $p$ \\
\hline Agravamento do estado de saúde desde o início da pandemia, $\mathrm{n}(\%)$ & $82(61)$ & $52(39)$ & 0,293 \\
\hline Teste positivo para COVID-19 & $2(50)$ & $2(50)$ & 0,227 \\
\hline Hospitalização por COVID-19 & $2(100)$ & $0(0)$ & 0,449 \\
\hline Morte de alguém próximo por COVID-19 & $21(84)$ & $4(16)$ & 0,055 \\
\hline Sintomas compatíveis com infeção & $5(50)$ & $5(50)$ & 0,975 \\
\hline Nunca ter saído de casa desde o início da pandemia & $102(51)$ & $99(49)$ & $0,000^{*}$ \\
\hline Agravamento da ansiedade desde o início da pandemia & $240(66)$ & $121(34)$ & 0,965 \\
\hline Agravamento da tristeza desde o início da pandemia & $198(64)$ & $113(36)$ & 0,498 \\
\hline Agravamento de problemas do sono desde o início da pandemia & $78(68)$ & $37(32)$ & 0,566 \\
\hline Adiamento de consultas devido à pandemia & $346(67)$ & $168(33)$ & 0,171 \\
\hline Recusa de consulta médicos devido à pandemia pelos serviços & $51(67)$ & $25(33)$ & 0,803 \\
\hline Abandono de cuidados de saúde pelo próprio & $73(66)$ & $37(34)$ & $37(34)$ \\
\hline Desemprego ou lay-off & $33(60)$ & $3(40)$ & $0,000^{*}$ \\
\hline Dificuldades em realizar pagamentos & $240(65)$ & $127(35)$ & 0,405 \\
\hline
\end{tabular}

${ }^{*}$ Resultados estatisticamente significativos $(p<0,05)$

\section{DISCUSSÃO}

A grande maioria dos participantes considera que o seu estado de saúde não se alterou, ainda que quase metade refira um atraso nos seus tratamentos médicos devido à pandemia. A grande maioria dos inquiridos deixou de visitar ou visitou com menor regularidade os seus familiares $\mathrm{e}$ quase metade não voltou a sair para caminhar como antes da pandemia, ou reduziu estes hábitos. A maioria dos inquiridos revelou, ainda, um aumento importante dos sintomas ansiosos e depressivos desde o início da pandemia.

Apenas uma minoria dos inquiridos teve um teste positivo para a SARS-CoV-2 ou apresentou sintomas compatíveis com esta infeção. A prevalência de infeção nesta faixa etária, até à data em questão (agosto 2020), é de $5,7 \%,{ }^{3} \mathrm{e}$ esta sub-representação de doentes com COVID-19 poderá dever-se ao facto de se tratar de uma amostra na comunidade com taxas inferiores de infeção quando comparadas com os idosos institucionalizados. Ainda sobre o impacto da pandemia na saúde física, quase metade dos inquiridos afirma ter verificado um atraso nos seus tratamentos médicos, com cerca de $12 \%$ dos inquiridos a afirmar ter desistido de algum cuidado médico por medo de serem infetados com o novo coronavírus. No entanto, paradoxalmente, a maioria dos inquiridos não percecionou uma alteração do seu estado de saúde. Sendo certo que está descrito na literatura internacional ${ }^{20,21}$ que $\mathrm{o}$ atraso no tratamento de diversas patologias, incluindo tratamentos urgentes, teve repercussão na mortalidade, esse impacto pode ser diferido no tempo e, portanto, não percecionado de forma imediata pela nossa amostra à data da resposta ao inquérito. 
Valeria, portanto, a pena, num horizonte a longo prazo, voltar a entrevistar esta amostra para se aferir se efetivamente foi percecionado um agravamento da sua saúde em consequência das limitações de acesso aos cuidados durante a pandemia.

Quase metade dos inquiridos não voltou a sair para caminhar ou reduziu estes hábitos. O sedentarismo tem um impacto sobejamente conhecido, tanto a nível da saúde física ${ }^{22}$ como mental. ${ }^{23}$ Neste último campo, sabe-se que existe uma associação importante entre o sedentarismo e a depressão nos mais velhos, sendo a atividade física uma estratégia preventiva e também curativa da depressão nos idosos. ${ }^{24} \mathrm{O}$ sedentarismo reduz a esperança média de vida e, perante uma redução abrupta da atividade física nas populações com idade avançada, verifica-se perda de massa muscular a um ritmo acelerado. ${ }^{25}$ Assim, nesta população idosa, a diminuição da atividade física pode comprometer a capacidade futura para a mesma e eventualmente a autonomia de cada indivíduo, de forma permanente.

A maioria dos inquiridos revelou aumento dos sintomas ansiosos e depressivos desde o início da pandemia, sendo este achado compatível com o de estudos internacionais. ${ }^{26}$ Tal pode dever-se a fatores já mencionados, como o aumento da solidão, menor socialização, diminuição da atividade física e até receio das consequências físicas que uma possível infeção possa ter. Aos mais velhos foi pedida especial cautela, muitas vezes com os bens essenciais levados a suas casas, o que se traduz num confinamento importante e grande alteração das suas rotinas, com um pano de fundo de incerteza. É importante ter também em consideração que $22 \%$ daqueles que estavam empregados perdeu o emprego devido à pandemia, e que mais de metade referiu dificuldades em continuar a pagar as suas despesas até ao final do mês, o que logicamente pode contribuir para o aumento de sintomas depressivos e ansiosos. ${ }^{27}$

A maioria dos inquiridos deixou de visitar os seus familiares, ou fê-lo com menor regularidade. Como sabemos, o isolamento social diminui os níveis de satisfação relativamente à vida, ${ }^{28} \mathrm{e}$ é um fator de risco para a saúde mental identificado de forma consistente nesta faixa etária. ${ }^{29}$ A população mais velha sente com maior impacto essas medidas em períodos de exigência de confinamento, por residir com frequência sozinha ou com outro idoso (nomeadamente por viuvez ou por ter filhos adultos já autónomos) e pela diminuição de autonomia por dificuldades físicas ou cognitivas,. De facto, $40 \%$ dos inquiridos referiu sentimentos de solidão agravados com o início da pandemia.

Várias limitações devem ser consideradas neste estudo. A amostra é apenas representativa dos maiores de 60 anos e não a partir dos 50 anos como em outros países europeus, sendo reduzido o número de participantes ativos nos quais se poderiam identificar maior impacto de medidas como o desemprego ou lay-off. Outra limitação é a inexistência de questionários ou escalas validadas de avaliação dos sintomas ansiosos ou depressivos ou de indicadores objetivos do estado de saúde. Contudo, apesar de não ter sido possível aplicar completamente a escala europeia de depressão geriátrica (escala Euro-D), tal como em outras vagas, foram, ainda assim, mantidas as questões sobre tristeza/depressão, ansiedade e problemas de sono.

\section{CONCLUSÃO}

Em conclusão, a pandemia de COVID-19 teve um impacto significativo nesta faixa etária, nomeadamente na redução do acesso aos cuidados de saúde, pelos próprios ou imposta, na diminuição da socialização e na deterioração de hábitos de vida, conduzindo a um desenvolvimento ou agravamento de sintomatologia ansiosa e depressiva.

Em relação a outros aspetos associados à pandemia, as mulheres referiram mais frequentemente agravamento do seu estado de saúde, tal como terem ficado mais em casa desde o início da pandemia, abandonarem mais frequentemente as consultas ou tratamentos em curso e terem sentido mais dificuldades em conseguir que os seus rendimentos fossem suficientes para cobrir as suas despesas até ao final do mês.

Mais estudos são necessários para avaliar o impacto a longo-prazo da pandemia de COVID-19 na faixa etária estudada, possivelmente incluindo também participantes institucionalizados, de modo a que se possa obter maior informação sobre o impacto global nesta população.

\section{CONTRIBUTO DOS AUTORES}

LN: Conceção do trabalho, análise e interpretação dos dados, redação do primeiro draft.

CC, PCP, BCR, TRS: Participação na escrita do primeiro draft, análise critica e aprovação final.

ADM: Recolha dos dados, revisão critica do trabalho estatístico e interpretação dos dados, revisão critica do manuscrito, aprovação final.

DTC: Revisão crítica do manuscrito, aprovação final

\section{PROTEÇÃO DE PESSOAS E ANIMAIS}

Os autores declaram que os procedimentos seguidos estavam de acordo com os regulamentos estabelecidos pelos responsáveis da Comissão de Investigação Clínica e Ética e de acordo com a Declaração de Helsínquia da Associação Médica Mundial atualizada em 2013.

\section{CONFIDENCIALIDADE DOS DADOS}

Os autores declaram ter seguido os protocolos do seu centro de trabalho acerca da publicação de dados.

\section{CONFLITOS DE INTERESSE}

Os autores declaram não ter conflitos de interesses relacionados com o presente trabalho.

\section{FONTES DE FINANCIAMENTO}

Este trabalho não recebeu qualquer tipo de suporte financeiro de nenhuma entidade no domínio público ou privado 


\section{REFERÊNCIAS}

1. Skoda EM, Bäuerle A, Schweda A, Dörrie N, Musche V, Hetkamp M, et al. Severely increased generalized anxiety, but not COVID-19-related fear in individuals with mental illnesses: a population based crosssectional study in Germany. Int J Soc Psychiatry. 2021;67:550-8.

2. Direção Geral da Saúde. Comunicado de casos de infeção por novo coronavírus (COVID19). [consultado 2021 mar 14]. Disponível em: https://covid19.min-saude.pt/wp-content/uploads/20.

3. Direção Geral da Saúde. Relatório de situação. [consultado 2021 mar 12]. Disponível em: https://covid19.min-saude.pt/wp-content/ uploads/2020/08/161_DGS_boletim_20200810.pdf.

4. O'Connor R, Hotopf $\bar{M}$, Worthman $\bar{C}$, Perry $H$, Tracey I, Wessely S, et al. Multidisciplinary research priorities for the COVID-19 pandemic authors' reply. Lancet Psychiatry. 2020;7:e44-5.

5. Rubin G, Wessely S. The psychological effects of quarantining a city. BMJ. 2020;368:m313.

6. Luo M, Guo L, Yu M, Jiang W, Wang H. The psychological and mental impact of coronavirus disease 2019 (COVID-19) on medical staff and general public - a systematic review and meta-analysis. Psychiatry Res. 2020;291:113190.

7. Bäuerle A, Teufel M, Musche V, Weismüller B, Kohler H, Hetkamp M, et al. Increased generalized anxiety, depression and distress during the COVID-19 pandemic: a cross-sectional study in Germany. J Public Health. 2020;42:672-8.

8. Choi E, Hui B, Wan E. Depression and anxiety in Hong Kong during COVID-19. Int J Environ Res Public Health. 2020;17:3740.

9. Jacobson N, Lekkas D, Price G, Heinz M, Song M, O'Malley A, et al. Flattening the mental health curve: COVID-19 stay-at-home orders are associated with alterations in mental health search behavior in the United States. JMIR Ment Health. 2020;7:e19347.

10. Brooks S, Webster R, Smith L, Woodland L, Wessely S, Greenberg N, et al. The psychological impact of quarantine and how to reduce it: rapid review of the evidence. Lancet. 2020;395:912-20.

11. Gloster A, Lamnisos D, Lubenko J, Presti G, Squatrito V, Constantinou M, et al. Impact of COVID-19 pandemic on mental health: an international study. PloS One. 2020;15:e0244809.

12. Guessoum S, Lachal J, Radjack R, Carretier E, Minassian S, Benoit L, et al. Adolescent psychiatric disorders during the COVID-19 pandemic and lockdown. Psychiatry Res. 2020;291:113264.

13. Pombo S, Câmara-Pestana P, Marinho ANF. How portuguese people deal with Covid-19 pandemic? Immediate impact of lockdown. Psicol Saúde Doenças. 2020;21:558-70.

14. Seifert A. The digital exclusion of older adults during the COVID-19 pandemic. J Gerontol Soc Work. 2020;63:674-6.

15. O'Driscoll M, Ribeiro Dos Santos G, Wang L, Cummings D, Azman A, Paireau J, et al. Age-specific mortality and immunity patterns of SARSCoV-2. Nature. 2021;590:140-5.
16. Venkatesh A, Edirappuli S. Social distancing in covid-19: what are the mental health implications? BMJ. 2020;369:m1379.

17. Börsch-Supan A. Survey of health, ageing and retirement in Europe (SHARE) wave 8. COVID-19 survey 1 interview date. Published online 2020. doi:10.6103/SHARE.W8CAINTDBETA.001

18. Börsch-Supan A. Survey of Health, Ageing and Retirement in Europe (SHARE) Wave 8. COVID-19 Survey 1. [consultado 2021 jun 17]. Disponível em: http://www.share-project.org/special-data-sets/sharecorona-survey.html.

19. Ouchi $Y$, Rakugi $H$, Arai $H$, Akishita M, Ito $H$, Toba $K$, et al. Redefining the elderly as aged 75 years and older: proposal from the Joint Committee of Japan Gerontological Society and the Japan Geriatrics Society. Geriatr Gerontol Int. 2017;17:1045-7.

20. Miyagami T, Uehara Y, Harada T, Watari T, Shimizu T, Nakamura A, et al. Delayed treatment of bacteremia during the COVID-19 pandemic. Diagnosis. 2021;8:327-32.

21. Czeisler M, Marynak K, Clarke K, Salah Z, Shakya I, Thierry J, et al. Delay or avoidance of medical care because of COVID-19-related concerns - United States, June 2020. MMWR Morb Mortal Wkly Rep. 2020;69:1250-7.

22. Santini Z, Jose P, York Cornwell E, Koyanagi A, Nielson L, Hinrichsen $\mathrm{C}$, et al. Social disconnectedness, perceived isolation, and symptoms of depression and anxiety among older Americans (NSHAP): a longitudinal mediation analysis. Lancet Public Health. 2020;5:e62-70.

23. Walker E, McGee R, Druss B. Mortality in mental disorders and global disease burden implications: a systematic review and meta-analysis. JAMA Psychiatry. 2015;72:334-41.

24. Ciucurel C, Iconaru El. The importance of sedentarism in the development of depression in elderly people. Procedia Soc Behav Sci. 2012;33:722-6.

25. Huber B, Schlichtiger J, Drey M, Steffen J, Brunner S. Change of the physical activity scale for the elderly (PASE) score after COVID-19 outbreak. J Sports Med Phys Fitness. 2021 (in press). doi: 10.23736/ S0022-4707.21.12453-3.

26. Machado-Lima A, Alonso A, Gozzo D, Zanca G, Brech G, Montiel J, et al. Self-perception of changes in routines in adults and older adults associated to social distancing due to COVID-19-A study in São Paulo, Brazil. Front Psychol. 2021;12:607559.

27. Brenner M, Bhugra D. Acceleration of anxiety, depression, and suicide: secondary effects of economic disruption related to COVID-19. Front Psychiatry. 2020;11:592467

28. Passos L, Prazeres F, Teixeira A, Martins C. Impact on mental health due to COVID- 19 pandemic: cross-sectional study in Portugal and Brazil. Int J Environ Res Public Health. 2020;17:6794.

29. Courtin E, Knapp M. Social isolation, loneliness and health in old age: a scoping review. Health Soc Care Community. 2017;25:799-812. 